\title{
Miedo a equivocarse y motivación autodeterminada en estudiantes adolescentes
}

\author{
Fear of failure and self-determined motivation among adolescent students
}

\section{Medo do fracasso e motivação auto-determinada entre estudantes adolescentes}

\author{
Yolanda Silveira Torregrosa y Juan Antonio Moreno Murcia
}

Universidad Miguel Hernández, Elche

\begin{abstract}
Resumen: El objetivo de este estudio fue analizar los efectos de la manipulación del clima motivacional, y del feedback positivo, verbal y no verbal, en relación con el miedo a equivocarse, la motivación intrínseca, y la importancia que le concede el estudiante a la Educación Física, teniendo como mediadores las necesidades psicológicas básicas. Se utilizó un diseńo cuasi experimental con grupo control no equivalente. La muestra estuvo compuesta por estudiantes de primer curso de Educación Secundaria, con edades entre 12 y 13 ańos $(M=12.14, D T=.42)$. En el grupo experimental $(n=20)$ se transmitió un clima tarea y un grupo control $(n=17)$ sobre el que no hubo manipulación. La intervención duró 30 sesiones de 50 minutos, con una frecuencia de dos sesiones semanales. Se midió antes y después de la misma. Los resultados revelaron un incremento en el grupo experimental en el feedback positivo, percepción de competencia, motivación intrínseca, en la concesión de importancia a la Educación Física, disminuyendo todas las variables del miedo a equivocarse. Por lo que la intervención fue efectiva ya que hubo mejoras en el grupo experimental en las variables motivacionales, viéndose disminuida la del miedo a fallar.

Palabras clave: Miedo a fallar; feedback; motivación intrínseca; Educación Física.

Abstract: The aim of this study was to analize effects of manipulating the motivational climate and positive verbal and nonverbal feedback in connection with the fear of failure, intrinsic motivation and the importance that students attribute to physical education, using psychological needs as mediators. In this study, we use pretest-posttest and quasi-experimental design with nonequivalent control group. The sample consisted of first-year secondary students, aged between 12 and $13(M=12.14, T D=.42)$ : an experimental
\end{abstract}

group $(n=20)$, assigned a mastery climate, and a control group $(n=17)$ where there was no manipulation. The intervention consisted of 30 class sessions of 50 minutes twice a week. It was measured before and at the end of the intervention. The results showed an increase in positive feedback, perceived competence, intrinsic motivation and the importance attributed to physical education, and a decrease in all the variables of fear of failure in the experimental group. Therefore, the intervention was effective since motivational variables improved, and decreased the fear of failure.

Key words: fear of failure; feedback; intrinsic motivation; physical education. Resumo: O objectivo deste estudo foi analisar os efeitos da manipulaçáo do clima motivacional, e do feedback positivo, verbal e não verbal, em relaçáo com o medo a se equivocar, a motivação intrinseca, e a importância que the concede o estudante à educação física, tendo como mediadores as necessidades psicológicas básicas. Utilizou-se um desenho cuasi experimental com grupo controle náo equivalente. A mostra esteve composta por estudantes de primeiro curso de Educaçấo Secundária, com idades entre 12 e 13 anos $(\mathrm{M}=$ 12.14 , DT $=.42)$. No grupo experimental $(\mathrm{n}=20)$ transmitiu-se um clima tarefa e um grupo controle $(n=17)$ sobre o que nấo teve manipulação. A intervenção durou 30 sessôes de 50 minutos, com uma frequência de duas sessốes semanais. Mediu-se dantes e após a mesma. Os resultados revelaram um incremento no grupo experimental no feedback positivo, percepção de concorrência, motivação intrínseca, na concessão de importância à educação física, diminuindo todas as variáveis do medo a se equivocar. Assim, a intervenção foi eficaz, pois houve melhorias no grupo experimental em variáveis motivacionais, vendo diminuiu o medo do fracasso.

Palavras-chave: Medo a falhar; feed-back; motivação intrínseca; educação física.

\section{Introducción}

Cada vez existe mayor interés en estudiar las consecuencias y patrones de conducta que ocurren en el estudiante de Educación Física tras la aplicación de un determinado clima motivacional en el aula mediante el desarrollo de estrategias de intervención (Barkoukis, Koidou, y Tsorbatzoudis, 2010; González-Cutre, Sicilia, y Moreno-Murcia, 2011; Moreno, Gómez, y Cervelló, 2010). La gran mayoría de estos estudios de intervención se basan en la propuesta de Ames (1992), los cuales hacen referencia a la manipulación de seis áreas de actuación y estrategias en torno al aprendizaje, conocidas por el acrónimo TARGET, en las que el feedback es una de las

Dirección para correspondencia [Correspondence address]: Juan Antonio Moreno Murcia. Centro de Investigación del Deporte. Universidad Miguel Hernández de Elche. Avenida de la Universidad, s/n. 03202 Elche, Alicante (Espańa). E-mail: j.moreno@umh.es estrategias utilizadas. Los resultados han mostrado que un clima que implique a la tarea favorece una mayor motivación intrínseca (González-Cutre et al., 2011; Moreno, Hernández, y González-Cutre, 2009), actitudes positivas, una mayor satisfacción en las clases (Tresure, 1993), mayor diversión y menor ansiedad (Barkoukis, Tsorbatzoudis, y Grouios, 2008), mayor voluntad para realizar tareas difíciles (Solomon, 1996) y menor miedo a fallar (Moreno-Murcia y Conte, 2011) siendo escasos los trabajos experimentales donde se contemplen modelos globales en relación al miedo a equivocarse en Educación Física. Por lo que este trabajo podría aportar información relevante en relación a como un clima motivacional que implica a la tarea, puede influir de forma positiva en las distintas variables motivacionales, disminuyendo las conductas de miedo y de evitación en los estudiantes de Educación Física. 
Una de las áreas más estudiadas y determinantes en la Educación Física, es el feedback que recibe el estudiante durante el proceso de enseñanza-aprendizaje, ya que el tipo de retroalimentación recibido y la forma en que se trasmite éste, no sólo va a permitir informar y mejorar la ejecución motriz del estudiante, sino que va a servir también para reforzar y motivar, mejorando así el clima socio-afectivo en el aula (Viciana, Cervelló, Ramírez, San-Matías, y Requena, 2003; Molina, Torres, y Miranda, 2008). Según los estudios de Koka y Hein (2001), el feedback verbal positivo es el que mejor predice la motivación intrínseca. En la misma línea, Amorose y Smith (2003), Koka y Hein (2003), Nicaise, Cogerino, Bois, y Amorose (2006) encontraron una relación positiva entre el feedback verbal positivo, la percepción de competencia y la motivación intrínseca. En relación al no verbal, hay que resaltar que los estudios son bastantes escasos, aunque no por ello menos importantes, ya que también se ha demostrado su relación positiva con la motivación intrínseca (Koka y Hein, 2005; Moreno-Murcia y Huéscar, 2012; Moreno-Murcia, Huéscar, Peco, Alarcón, y Cervelló, 2013; Vallejo y Plested, 2008).

La propia naturaleza de las actividades desarrolladas en las clases de Educación Física, crea un escenario único que permite al estudiante mostrar ante sus iguales, su nivel de habilidad motriz, pudiendo crear en ocasiones cierto grado de inseguridad, ansiedad, estrés y conductas de evitación en los estudiantes menos hábiles, como consecuencia del miedo a cometer errores y pasar vergüenza por lo que puedan decir o pensar los demás. Según Zuckerman y Tsai (2005), este miedo a equivocarse y las excusas para no realizar la tarea, van a proteger la autoestima a corto plazo, pero a la larga van a afectar mucho a la persona, dando lugar a una peor salud y bienestar, bajo nivel de competencia, de motivación intrínseca y de estado de ánimo.

Según la teoría de la autodeterminación de Deci y Ryan $(1985,2000)$, generar un entorno que satisfaga las tres necesidades psicológicas básicas del participante (percepción de competencia, autonomía y relación con los demás), llevaría a un aumento de la motivación intrínseca, mientras que la frustración estaría asociada a consecuencias negativas en la salud psicológica o bienestar personal del practicante, dando lugar a una menor motivación autodeterminada (GarcíaCalvo, Sánchez, Leo, Sánchez, y Amado, 2011). En relación al miedo a equivocarse, Conroy, Poczwardowski, y Henschen (2001) determinaron que las percepciones del fallo van a depender más de cómo se satisfagan las necesidades psicológicas básicas de los practicantes, que por el rendimiento objetivo y la competencia. De esto se deduce que experimentar una motivación más autodeterminada, mediante la satisfacción de estas tres necesidades psicológicas básicas, podría disminuir en el estudiante los sentimientos negativos de estrés, tensión, ansiedad, y conductas de evitación que tienen relación con el miedo a equivocarse (Conroy, Coastworth, y Kaye, 2007; Moreno-Murcia y Conte, 2011). De manera que este estado de motivación o de implicación del estudiante más autodeterminado, va a dar lugar a que le dé una mayor importancia a la asignatura de Educación Física (Moreno, y Llamas, 2007; Moreno, Zomeńo, Marín, Ruiz, y Cervelló, 2013), y presente una actitud positiva hacía la misma (Florence, 1991; Moreno y Cervelló, 2003), y como consecuencia de ello, muestre mayor compromiso, adherencia a la práctica deportiva, esfuerzo y persistencia (Ferrer-Caja y Weiss, 2000; Moreno, Cervelló, y González-Cutre, 2007; Standage et al., 2003).

Por último, hay que decir que el tipo de feedback que percibe el estudiante respecto al docente tiene un efecto sobre el grado de motivación hacía la tarea que está practicando, y también puede influir en su percepción de éxito o fracaso. Según esto, le llevaría a enfrentarse a tareas que supongan un mayor reto y no presentar conductas de evitación. Por todo ello, el objetivo de este trabajo ha sido analizar los efectos de la manipulación del clima motivacional, prestando especial atención al feedback, verbal y no verbal, en relación al miedo a equivocarse, y cómo influye sobre el grado de motivación intrínseca, la importancia que le concede el estudiante a la Educación Física, teniendo como mediadores las necesidades psicológicas básicas. Se espera que un clima motivacional que implica a la tarea junto a una mayor trasmisión de feedback positivo se relacione de forma negativa con el miedo a equivocarse y de forma positiva con mediadores psicológicos, dando lugar a una mayor motivación intrínseca, y a un incremento en la importancia que el estudiante otorga a la Educación Física.

\section{Método}

\section{Participantes}

Finalmente participaron un total de 37 estudiantes de Educación Física pertenecientes a dos clases diferentes de primer curso de Educación Secundaria Obligatoria de un mismo centro educativo, de los cuales 22 eran chicos y 15 chicas, con edades comprendidas entre los 12 y 13 años $(M=12.14, D T=$ .42). Una de las clases fue el grupo experimental $(n=20), 11$ de los cuales eran chicos y 9 chicas y la otra el grupo control $(n=17)$, de los cuales 11 eran chicos y 6 chicas.

Como consecuencia de errores y/o omisiones en las respuestas a la hora de cumplimentar los instrumentos de evaluación, y la ausencia de algunos participantes en dicho momento, se procedió a su eliminación.

\section{Instrumentos}

Feedback positivo. Se utilizaron las dimensiones de feedback general positivo y no verbal positivo de la escala Perceptions of Teacher's Feedback Scale (Koka y Hein, 2003) validada al contexto español por Moreno-Murcia y Huéscar (2012). Los 
factores estaban compuestos por tres ítems (e.g. "Mi trabajo es con frecuencia animado/alentado por el docente"), y por cuatro ítems (e.g. "El docente me elogia a pesar de que no lo merezca”), respectivamente. Estos ítems estaban encabezados por la frase "En mis clases de Educación Física", que se respondían con una escala Likert de 1 (Falso) a 5 (Verdadero). La consistencia interna reveló un valor alfa de Cronbach de $.78 \mathrm{y}$ .75 en el pre-test y de .76 y .78 en el pos-test, respectivamente.

Miedo a equivocarse. Se utilizó la versión larga (PFAI; 25 ítems) del Inventario de Evaluación del Error en el Rendimiento (PFAI) de Conroy et al. (2002) que evalúa el error en el rendimiento, y validado al contexto español por MorenoMurcia y Conte (2011). La escala consta de 25 ítems, agrupados en cinco factores: miedo de experimentar vergüenza (e.g. "Cuando me equivoco, me da vergüenza si los demás están allí para verlo"), miedo a la devaluación de uno mismo (e.g. "Cuando no tengo éxito, me siento menos valioso que cuando tengo éxito"), miedo de tener un futuro incierto (e.g. "Cuando me equivoco, creo que mis planes para el futuro cambiarán”), miedo de perder el importante interés de los demás (e.g. "Cuando no tengo éxito, algunas personas no se muestran interesadas por mí”), y el temor de perturbar a otros importantes (e.g. "Cuando me equivoco, esto disgusta a la gente que me importa"). Las respuestas son cerradas y se responden a través de una escala tipo Likert, que va de 1 (No lo creo nada) a 5 (Lo creo al 100\%). Estos ítems estaban encabezados por la frase "En las clases de educación física". La consistencia interna reveló un valor alfa de Cronbach de .85, $.67, .83, .87$ y .90 , respectivamente en el pre-test y de $.81, .72$, $.89, .85$ y .84 en el pos-test.

Necesidades psicológicas básicas. Se utilizó la Psychological Need Satisfaction in Exercise Scale (PNSE) de Wilson, Rogers, Rodgers, y Wild (2006) validada al contexto español por Moreno-Murcia, Huéscar, y Cervelló (2012). La PNSE utiliza 18 ítems, seis para evaluar cada una de las necesidades: competencia (e.g. "Tengo confianza para hacer los ejercicios más desafiantes"), autonomía (e.g. "Creo que puedo tomar decisiones en mis clases de Educación Física”), y relación con los demás (e.g. "Me siento unido a mis compañeros de clase porque ellos me aceptan como soy). La sentencia previa fue "En mis clases de Educación Física" y las respuestas son recogidas en una escala tipo Likert, cuyo rango de puntuación oscilaban entre 1 (Falso) y 6 (Verdadero). La consistencia interna reveló un valor alfa de Cronbach de .89, .70 y .77, respectivamente en el pre-test y de $.94, .78$ y .79 , respectivamente en el pos-test.

Motivación intrínseca. Se empleó el factor motivación intrínseca de la Escala del Locus Percibido De Causalidad (PLOC Scale) de Goudas, Biddle, y Fox (1994), validado al contexto español por Moreno, González-Cutre, y Chillón (2009). Este factor, encabezado por el enunciado "Participo en esta clase de Educación Física”, está compuesto por cuatro ítems (e.g.
"Porque disfruto aprendiendo nuevas habilidades") que se responden con una escala tipo Likert que va de 1 (Totalmente en desacuerdo) a 7 (Totalmente de acuerdo). La consistencia interna reveló un valor alfa de Cronbach de .83 en el pre-test y de .89 en el pos-test.

Importancia de la Educación Física. Se midió la variable importancia y utilidad concedida por el estudiante a la Educación Física a través de la Escala de Importancia de la Educación Física (IEF) (Moreno et al., 2006). Está formada por tres ítems agrupados en un solo factor: "Considero importante recibir clases de Educación Física", "Comparado con el resto de asignaturas, creo que la Educación Física es una de las más importantes" y "Creo que las cosas que aprendo en Educación Física me serán útiles en mi vida”. Los estudiantes debían responder en una escala tipo Likert con un rango de puntación que oscilaba desde 1 (Totalmente en desacuerdo) a 4 (Totalmente de acuerdo). Estos ítems estaban encabezados por la frase: "Respecto a las clases de Educación Física". La consistencia interna obtenida en el pre-test y el pos-test fue de .74 y de .71 , respectivamente.

\section{Diseño y Procedimiento}

Se utilizó un diseño cuasi-experimental (pretest-postest) con grupo control no equivalente (Campbell y Stanley, 1966), puesto que al tratarse de grupos de clases ya establecidos por el centro de enseñanza no se podía respetar la aleatorización. Se escogieron dos grupos de primer curso de Educación Secundaria Obligatoria, uno experimental de 20 estudiantes y otro control de 17 estudiantes. Cada grupo tuvo un docente diferente. En el grupo experimental el docente transmitió un clima tarea, centrado en la trasmisión de feedback positivo y en la manipulación de las áreas target de Ames (1992), mientras que en el grupo control no hubo ningún tipo de manipulación. La intervención fue realizada entre los meses de enero y mayo de 2012, con un total de 30 sesiones de 50 minutos de duración, y con una frecuencia de dos sesiones semanales. Dicha intervención englobó tres unidades didácticas: acrogimnasia, juegos modificados y malabares. En el grupo control los contenidos impartidos fueron los mismos.

Para llevar a cabo la investigación se solicitó permiso a la dirección del centro, estando el proyecto aprobado por el claustro de profesorado y por el consejo escolar. Todo el alumnado participó voluntariamente en el estudio, quedando informados del mismo sus padres, madres o tutores. Antes de iniciar la intervención, se administraron a ambos grupos los diferentes cuestionarios descritos en el apartado de medidas. Se informó a los estudiantes de forma breve acerca del objetivo del estudio y de cómo rellenar los cuestionarios, insistiendo en el anonimato de los mismos y de responder de forma individual y con sinceridad. El tiempo requerido para cumplimentarlos fue de 30 minutos aproximadamente. 
Previo a la intervención, fue necesario formar al docente del grupo experimental para poder transmitir un clima tarea de forma adecuada, incidiendo especialmente en la trasmisión de feedback positivo, tanto de forma verbal como no verbal. Para ello fue necesaria la formación del docente, asistiendo a diferentes seminarios de formación en los que se explicaba cómo manipular las clases para fomentar un clima tarea. Estos seminarios estaban centrados en describir las premisas establecidas por Ames (1992) bajo las siglas target (Tarea, Autoridad, Reconocimiento, Grouping o agrupación, Evaluación y Tiempo). El comportamiento del docente fue analizado al inicio de la intervención (durante las primeras cinco sesiones) a través de una adaptación del Coaching Behavior Assessment System (CBAS; Smith, Smoll, y Hunt, 1977). Este instrumento medía 12 categorías de la conducta del docente organizadas en dos dimensiones: conducta general del docente y comportamiento del docente cuando respondía al rendimiento del estudiante. La primera dimensión incluía la organización, información técnica, comunicación general e instrucciones de ánimo de forma global. La segunda dimensión evaluaba las respuestas del docente ante ejecuciones correctas y ante errores, incluyendo el feedback, palabras de ánimo, castigos y ausencia de respuesta. Se utilizó este instrumento porque en investigaciones previas (González-Cutre, Sicilia, y Moreno, 2011; Goudas, Biddle, Fox, y Underwood, 1995; Wallhead y Ntoumanis, 2004) fue utilizado con éxito para medir la conducta del docente en diferentes programas de intervención centrados en la tarea, y así garantizarnos de que en cada sesión se estaba trasmitiendo un clima tarea.

En el análisis observacional participaron el investigador principal y una persona ajena a la investigación. Ambos fueron entrenados previamente para registrar la actuación del docente en relación con el clima tarea. Fueron necesarias cinco sesiones de entrenamiento hasta conseguir una fiabilidad inter e intra-observadores del 90\%. Para evaluar la fiabilidad de la intervención se establecieron las siguientes dimensiones: el refuerzo positivo verbal y no verbal, el ánimo, el feedback técnico, el mantenimiento del control, la instrucción técnica de forma general, la instrucción de ánimo de forma general, la organización y la comunicación general. Presentando el grupo experimental una intervención en clima tarea de un $91.48 \%$.

\section{Análisis de datos}

Antes de comenzar con todos los análisis, fue necesario conocer la existencia de diferencias entre ambos grupos. Para ello se realizó un análisis diferencial. Tras la intervención, para conocer las diferencias intra-grupo se realizó una prueba $\mathrm{t}$ para muestras relacionadas, y otro análisis diferencial para conocer las diferencias inter-grupo. Los diferentes análisis se llevaron a cabo con el paquete estadístico SPSS 21.0.

\section{Resultados}

\section{Análisis preliminar}

En primer lugar, para comprobar la homogeneidad de ambos grupos antes de la intervención, se realizó un análisis diferencial, considerando como variables dependientes (feedback no verbal positivo y verbal positivo, miedo general, miedo de experimentar vergüenza, miedo a la devaluación de uno mismo, a tener un futuro incierto, a perder el interés de otros, a perturbar a otros importantes, competencia, autonomía, relación con los demás, motivación intrínseca, e importancia de la Educación Física) y como factor fijo (el grupo y el sexo) no encontrándose diferencias a nivel multivariado en relación al grupo (Lambda de Wilks $\left.=.23, F(22,14)=2.19, p>.05, \eta^{2}=.78\right)$, aunque los posteriores análisis reflejaron diferencias en las variables de miedo general $\left(F=5.32, p<.05, \eta^{2}=.13\right)$, miedo de experimentar vergüenza $\left(F=6.97, p<.01, \eta^{2}=.17\right)$, miedo a la devaluación de uno mismo $\left(F=4.670, p<.05, \eta^{2}=.12\right)$, miedo a perder el interés de otros $\left(F=6.52, p<.05, \eta^{2}=.16\right)$, miedo a perturbar a otros importantes $\left(F=5.88, p<.05, \eta^{2}=.14\right)$, donde el grupo experimental presentó puntuaciones mayores que el grupo control, no encontrándose diferencias según el sexo (Tabla 1).

Tabla 1. Análisis diferencial en el Pre-test.

\begin{tabular}{llllllll}
\hline & \multicolumn{3}{l}{ Grupo experimental } & \multicolumn{2}{c}{ Grupo control } & & \\
\hline & $M$ & $D T$ & $M$ & $D T$ & $F$ & $p$ & $\eta^{2}$ \\
\hline Feedback no verbal positivo & 3.21 & 1.00 & 2.81 & .73 & 1.89 & .18 & .05 \\
Feedback verbal positivo & 3.57 & 1.32 & 3.98 & 1.10 & 1.05 & .31 & .03 \\
Miedo general & 2.14 & .93 & 1.56 & .48 & 5.32 & .03 & .13 \\
Miedo de experimentar vergüenza & 2.66 & 1.05 & 1.88 & .67 & 6.97 & .01 & .17 \\
Miedo a la devaluación de uno mismo & 2.19 & .90 & 1.65 & .55 & 4.67 & .04 & .12 \\
Miedo a tener un futuro incierto & 1.73 & .86 & 1.37 & .80 & 1.74 & .20 & .05 \\
Miedo a perder el interés de otros & 2.08 & 1.06 & 1.35 & .55 & 6.52 & .02 & .16 \\
Miedo a perturbar a otros importantes & 2.03 & .89 & 1.46 & .42 & 5.88 & .02 & .14 \\
Competencia & 4.68 & 1.01 & 5.09 & .89 & 1.72 & .20 & .05 \\
\hline
\end{tabular}




\begin{tabular}{lccccccc}
\hline & \multicolumn{3}{c}{ Grupo experimental } & \multicolumn{2}{c}{ Grupo control } & & \\
\hline & $M$ & $D T$ & $M$ & $D T$ & $F$ & $p$ & $\eta^{2}$ \\
\hline Autonomía & 3.79 & .84 & 4.06 & .98 & .81 & .37 & .02 \\
Relación con los demás & 4.80 & .80 & 4.86 & .87 & .05 & .82 & .00 \\
Motivación intrínseca & 6.00 & 1.04 & 6.15 & .81 & .22 & .64 & .01 \\
Importancia Educación Física & 3.20 & .45 & 3.47 & .47 & 3.17 & .08 & .08 \\
\hline
\end{tabular}

\section{Efecto de la intervención}

Para conocer los efectos de la intervención en el grupo experimental se realizó una prueba t para muestras relacionadas. El análisis de los datos reflejó un incremento en su puntuación en todas las variables en el grupo experimental, encontrándose diferencias en el feedback no verbal positivo $(p<.05)$, feedback verbal positivo $(p<.05)$, miedo general $(p<.01)$, miedo de experimentar vergüenza $(p<.01)$, miedo a la de- valuación de uno mismo $(p<.05)$, miedo a tener un futuro incierto $(p<.01)$, miedo a perder el interés de otros $(p<.05)$, miedo a perturbar a otros importantes $(p<.01)$, percepción de competencia $(p<.01)$, motivación intrínseca $(p<.05)$, e importancia de la Educación Física $(p<.05)$. De igual forma en el grupo control se realizó la misma prueba donde solo se encontraron diferencias en el miedo a la devaluación de uno mismo $(p<.05)$, donde los valores se vieron ligeramente incrementados tras la intervención (Tabla 2).

Tabla 2. Diferencias entre la Toma Pre y Post Test en Grupo Experimental y Control.

\begin{tabular}{|c|c|c|c|c|c|c|c|c|c|}
\hline & & \multicolumn{4}{|c|}{ Grupo experimental } & \multicolumn{4}{|c|}{ Grupo control } \\
\hline & & $M$ & $D T$ & $t$ & $p$ & $M$ & $D T$ & $t$ & $p$ \\
\hline \multirow[t]{2}{*}{ Feedback no verbal positivo } & Pre & 3.21 & 1.00 & -2.22 & .04 & 2.81 & .73 & -.11 & .91 \\
\hline & Post & 3.76 & .58 & & & 2.84 & .80 & & \\
\hline \multirow[t]{2}{*}{ Feedback verbal positivo } & Pre & 3.57 & 1.32 & -1.89 & .04 & 3.98 & 1.10 & 1.05 & .31 \\
\hline & Post & 4.15 & .46 & & & 3.65 & .79 & & \\
\hline \multirow[t]{2}{*}{ Miedo general } & Pre & 2.14 & .93 & 2.94 & .01 & 1.56 & .48 & .00 & 1.00 \\
\hline & Post & 1.50 & .51 & & & 1.56 & .75 & & \\
\hline \multirow[t]{2}{*}{ Miedo de experimentar vergüenza } & Pre & 2.66 & 1.05 & 3.67 & .00 & 1.88 & .67 & .00 & 1.00 \\
\hline & Post & 1.79 & .62 & & & 1.88 & .85 & & \\
\hline \multirow[t]{2}{*}{ Miedo a la devaluación de uno mismo } & Pre & 2.19 & .90 & 2.49 & .02 & 1.65 & .55 & -2.33 & .03 \\
\hline & Post & 1.71 & .58 & & & 2.06 & .78 & & \\
\hline \multirow[t]{2}{*}{ Miedo a tener un futuro incierto } & Pre & 1.73 & .86 & 2.97 & .01 & 1.37 & .80 & -.77 & .45 \\
\hline & Post & 1.17 & .33 & & & 1.47 & .99 & & \\
\hline \multirow[t]{2}{*}{ Miedo a perder el interés de otros } & Pre & 2.08 & 1.06 & 2.95 & .01 & 1.35 & .55 & -.83 & .42 \\
\hline & Post & 1.44 & .50 & & & 1.46 & .79 & & \\
\hline \multirow[t]{2}{*}{ Miedo a perturbar a otros importantes } & Pre & 2.03 & .89 & 3.41 & .00 & 1.46 & .42 & -.76 & .46 \\
\hline & Post & 1.37 & .46 & & & 1.59 & .68 & & \\
\hline \multirow[t]{2}{*}{ Competencia } & Pre & 4.68 & 1.01 & -3.96 & .00 & 5.09 & .89 & .99 & .34 \\
\hline & Post & 5.42 & .76 & & & 4.89 & .99 & & \\
\hline \multirow[t]{2}{*}{ Autonomía } & Pre & 3.79 & .84 & -.04 & .97 & 4.06 & .98 & 1.89 & .08 \\
\hline & Post & 3.80 & 1.11 & & & 3.51 & .96 & & \\
\hline \multirow[t]{2}{*}{ Relación con los demás } & Pre & 4.80 & .80 & -.54 & .60 & 4.86 & .87 & -.37 & .71 \\
\hline & Post & 4.90 & .79 & & & 4.92 & .86 & & \\
\hline \multirow[t]{2}{*}{ Motivación intrínseca } & Pre & 6.00 & 1.04 & -1.79 & .04 & 6.15 & .81 & .89 & .38 \\
\hline & Post & 6.76 & .66 & & & 5.88 & 1.27 & & \\
\hline \multirow[t]{2}{*}{ Importancia Educación Física } & Pre & 3.20 & .45 & -2.14 & .04 & 3.47 & .47 & 1.99 & .06 \\
\hline & Post & 3.47 & .37 & & & 3.20 & 1.27 & & \\
\hline
\end{tabular}


Para comprobar las diferencias entre ambos grupos al finalizar la intervención, se realizó el mismo procedimiento que en el análisis preliminar. No se encontraron diferencias a nivel multivariado (Lambda de Wilks $=.25, F(22,14)=1.87, p$ $\left.>.05, \eta^{2}=.75\right)$, existiendo diferencias a nivel univariado en las variables de feedback no verbal positivo $(F=16.35, p<.01$, $\left.\eta^{2}=.32\right)$, feedback verbal positivo $\left(F=5.82, p<.05, \eta^{2}=\right.$ $.14)$, percepción de competencia $\left(F=3.31, p<.05, \eta^{2}=.09\right)$ y motivación intrínseca $\left(F=3.18, p<.05, \eta^{2}=.08\right)$ a favor del grupo experimental (Tabla 3$)$.

Tabla 3. Análisis diferencial en el Post-test.

\begin{tabular}{|c|c|c|c|c|c|c|c|}
\hline \multicolumn{8}{|l|}{ Grupo experimental Grupo control } \\
\hline & $M$ & $D T$ & $M$ & $D T$ & $F$ & $p$ & $\eta^{2}$ \\
\hline Feedback no verbal positivo & 3.76 & .58 & 2.84 & .80 & 16.35 & .00 & .32 \\
\hline Feedback verbal positivo & 4.15 & .46 & 3.65 & .79 & 5.82 & .02 & .14 \\
\hline Miedo general & 1.50 & .51 & 1.56 & .75 & .10 & .76 & .00 \\
\hline Miedo de experimentar vergüenza & 1.79 & .62 & 1.88 & .85 & .14 & .71 & .00 \\
\hline Miedo a la devaluación de uno mismo & 1.71 & .58 & 2.06 & .78 & 2.40 & .13 & .06 \\
\hline Miedo a tener un futuro incierto & 1.17 & .33 & 1.47 & .99 & 1.68 & .20 & .05 \\
\hline Miedo a perder el interés de otros & 1.44 & .50 & 1.46 & .79 & .01 & .93 & .00 \\
\hline Miedo a perturbar a otros importantes & 1.37 & .46 & 1.59 & .68 & 1.36 & .25 & .04 \\
\hline Competencia & 5.42 & .76 & 4.89 & .99 & 3.31 & .04 & .09 \\
\hline Autonomía & 3.80 & 1.11 & 3.51 & .96 & .73 & .40 & .02 \\
\hline Relación con los demás & 4.90 & .79 & 4.92 & .86 & .01 & .94 & .00 \\
\hline Motivación intrínseca & 6.76 & .66 & 5.88 & 1.27 & 3.18 & .04 & .08 \\
\hline Importancia Educación Física & 3.47 & .37 & 3.20 & .71 & 2.23 & .14 & .06 \\
\hline
\end{tabular}

\section{Discusión}

El objetivo de este trabajo fue analizar los efectos de la manipulación del clima motivacional, prestando especial atención al feedback positivo, verbal y no verbal, en relación al miedo a equivocarse, y su relación sobre el grado de motivación autodeterminada, la importancia que le concede el estudiante a la Educación Física, tomando como base la teoría de la autodeterminación. Tras la intervención del docente, todas las diferencias encontradas inicialmente se eliminaron, llegando a mejorar en el post-test al grupo control en las variables de feedback positivo verbal y no verbal, percepción de competencia y en la motivación intrinseca. Por lo que parece que la intervención del docente en la trasmisión de un clima motivacional que implique a la tarea, con un mayor refuerzo de feedback positivo, fue efectivo.

A pesar de que hubo una mejora en el grupo experimental en todas las variables tras la intervención, no todas fueron significativas. De manera que, los resultados obtenidos mostraron que un clima que implicaba a la tarea con un fuerte refuerzo de feedback positivo, dio lugar a una mayor percepción de feedback positivo verbal y no verbal, mayor competencia, mayor motivación intrinseca y mayor concesión de importancia a la asignatura de Educación Física y un menor miedo a equivocarse, no estableciéndose relación alguna con la percepción de autonomía y con la relación con los demás.

Partiendo de que numerosas investigaciones de corte ex- perimental han venido demostrado que un clima motivacional más orientado a la tarea favorece patrones conductuales y afectivos más positivos (Barkoukis et al., 2008; Digelidis et al., 2003; González-Cutre et al., 2011; Morgan y Carpenter, 2002), dando lugar a una mayor persistencia, esfuerzo, interés, diversión, satisfacción y mayor motivación intrínseca, se puede decir, que el feedback se presenta como elemento clave dentro de la creación del clima motivacional. Este permite al docente influir en los estudiantes durante el proceso de enseñanza y aprendizaje, en su percepción de éxito y fracaso (Carreiro da Costa, 1989).

En relación con el miedo a equivocarse y partiendo de que existen estudios que determinan que un clima que implica al ego se encuentra relacionado positivamente con la afectividad negativa y sentimientos de presión (Halliburton y Weiss, 2002; Krane et al., 1997; Theeboom et al., 1995), y con una mayor ansiedad (Ommundsen, 2001), también podría favorecer conductas de evitación y sentimientos de miedo a cometer errores en el estudiante. En nuestro estudio los resultados mostraron que el clima motivacional tarea parece tener una relación negativa con el miedo a equivocarse. Encontramos trabajos en el contexto deportivo, que indican que un clima motivacional que implica tarea se relaciona negativamente con el miedo a cometer errores (Moreno-Murcia y Conte, 2011). De ahí la importancia de desarrollar un clima motivacional tarea y no exclusivamente ego, para evitar sentimientos negativos de es- 
trés, ansiedad, malestar y de miedo a equivocarse, y generar en los estudiantes patrones de conducta más adaptativos.

Desde el punto de vista de la teoría de la autodeterminación (Deci y Ryan, 1985, 2000), el clima motivacional es considerado un factor social que va a influir sobre la motivación a través de la satisfacción de las tres necesidades psicológicas básicas (competencia, autonomía y relación con los demás). Autores como Ntoumanis y Biddle (1999) y Vallerand (2007), determinaron que un clima motivacional que implica a la tarea podía satisfacer las necesidades de competencia, autonomía y relación con los demás, asociándose con formas de motivación más autodeterminadas, las cuales conseguirán que el estudiante presente una mayor actitud positiva hacía la Educación Física (Florence, 1991; Moreno y Cervelló, 2003) y por tanto le pueda conceder una mayor importancia a esta asignatura (Moreno y Llamas, 2007; Moreno et al., 2013).

A pesar de que hubo una mejora en el grupo experimental en todas las variables tras la intervención, no todas fueron significativas. Podemos concluir que favorecer un clima motivacional que implique a la tarea con una mayor trasmisión de feedback positivo dio lugar a una disminución de miedo a equivocarse, así como una mayor percepción de competencia, mayor motivación intrínseca y conceder una mayor importancia a la Educación Física por parte de los estudiantes.

Como posibles limitaciones de este estudio, señalar el reducido número de muestra, la existencia de solo un grupo control y experimental, y el bajo tamaño del efecto obtenido en la intervención. Por lo que, en futuros estudios sería interesante ampliar la muestra, estableciendo más grupos experimentales y controles, así como plantear estudios de carácter longitudinal, lo que aportaría nuevos resultados sobre las consecuencias que tiene la manipulación del clima motivacional y el feedback utilizado por el docente, en relación al miedo a equivocarse de los estudiantes, y el grado de motivación.

\section{Aplicaciones prácticas}

Las experiencias negativas en las clases de Educación Física llevan a los estudiantes a sentirse menos competentes, de manera que aquellos estudiantes que temen sufrir las consecuencias del fallo van a tratar de evitar situaciones de logro. En este sentido, el fallo en sí no tendría connotaciones negativas si no fuera por la valoración social y las consecuencias aversivas que experimenta el estudiante en su autoestima. Por todo ello, el docente juega un papel importante en la creación de un clima de aula adecuado, que permita el desarrollo de conductas más autodeterminadas (mayor disfrute, bienestar, motivación intrínseca, superación, placer, etc.) y disminuya las conductas de evitación y de miedo a cometer errores en los estudiantes.

Con el fin de lograr una transferencia social de esta investigación, se presentan algunas de las estrategias motivaciona- les y recursos metodológicos que se han empleado durante la intervención en el grupo experimental, las cuales se apoyan en distintos trabajos de investigación (Moreno-Murcia, Conte, Silveira, y Ruiz, 2014; Moreno-Murcia, Silveira, y Conte, 2013; Silveira, 2013).

\section{Estrategias para favorecer la transmisión de la información y de feedback}

Es fundamental proporcionar una retroalimentación positiva a los estudiantes, y hacerles ver que se puede mejorar si uno se esfuerza en ello, animarles a seguir experimentando, lo que favorecerá la disminución de la ansiedad, el estrés y del miedo a cometer errores. El docente intentará animar al estudiante tanto mediante el lenguaje verbal (¡Bravo!, ¡Bien!, ¡Ánimo!, ¡Correcto!, ¡Eso es lo que buscamos!, ¡Lo has pillado muy bien!), como con el no verbal (señal de OK, guiño de ojo, pulgar arriba, asentir con la cabeza, etc.). Utilizar planillas de observación donde se valoren los aspectos técnicos de una forma cualitativa, no castigar nunca por los errores, ya que forman parte de todo proceso de aprendizaje. Evitar comentarios del tipo: “'Vamos, que eres al único al que no le sale!, "mira como lo hace tu compañero, a ver si aprendes de él". "Él está saltando ya más que tú, a ver si espabilas".

\section{Estrategias para favorecer la percepción de competencia}

Promover metas orientadas al proceso, como por ejemplo, plantear tareas con un cierto nivel de dificultad, adaptadas al nivel de cada estudiante, para que todos puedan realizar las tareas propuestas y sentirse competentes. Pedir permiso para que un estudiante haga la demostración. Es importante que el practicante no se sienta presionado ya que podría aumentar su sentimiento de ansiedad, estrés y de miedo hacia la actividad. Reconducir aquellas expresiones de los estudiantes, como "yo no sé hacer esto", "a mi no me sale", dando orientaciones del tipo "aqui venimos para aprender", "cada uno trabaja a su ritmo, no tenemos prisa”. Transmitir el deseo de observar las mejoras personales y no obtener grandes marcas. Evitar las evaluaciones cuantitativas, de forma exclusiva, centrándose en la observación sistemática de las conductas de los participantes, tales como el espíritu cooperativo, solidaridad, ilusión por participar, valoración de su propia realización corporal y la aceptación de sus limitaciones y de la de los demás.

\section{Estrategias para favorecer la percepción de autonomía}

Proponer tareas más participativas, es importante que los estudiantes reflexionen y sean conscientes de su progreso personal y mejora, que puedan decidir acerca de las normas, recompensas, castigos, etc., al inicio del curso, así como hacerles participar en la organización de las tareas propuestas 
en la clase. Además, es interesante proponer actividades que favorezcan el descubrimiento y resolución de problemas, proporcionar feedback interrogativo. Así mismo, sería conveniente implicar al participante en su propia evaluación, permitiéndole opinar sobre su propia acción motriz, actitudes o nuevos conocimientos.

\section{Estrategias para favorecer la percepción de relación con los demás}

El docente tiene que saber contagiar el entusiasmo que él tiene sobre el contenido de enseñanza, la profesión docente y las propias relaciones con los participantes, por tanto es fundamental llegar a clase con buen humor y mostrar entusiasmo por lo que se hace, ser receptivo a los sentimientos y emociones de los estudiantes. Favorecer diferentes formas de agrupamiento (atendiendo al número, características de los participantes, etc.) de manera que trabajen cada vez con distintos compañeros, lo que mejorará las relaciones personales. Intentar evitar, en la medida de lo posible, que se formen grupos siguiendo criterios de competencia a la hora de elegir los compañeros. Evitar realizar comparaciones entre los practicantes, ya que fomenta la competitividad, creando situaciones de estrés y ansiedad, que dañan las relaciones dentro del grupo, aumentando el miedo al fallo.

Con estas estrategias se pretender satisfacer las necesidades de competencia, autonomía, y relación con los demás de los estudiantes, con el fin de que no se sientan frustrados, y logren altos niveles motivación hacia la tarea.

\section{Referencias}

1. Ames, C. (1992). Achievement Goals, Motivational Climate and Motivational Processes. En G. C. Roberts (Ed.), Motivation in Sport and Exercise (pp. 161-176). Champaign, IL: Human Kinetics.

2. Amorose, A. J., y Smith, P. (2003). Feedback as a source of physical competence information: Effects of age, experience and type of feedback. Journal of Sport and Exercise Psychology, 25, 341-359.

3. Barkoukis, V., Tsorbatzoudis, H., y Grouios, G. (2008). Manipulation of motivational climate in physical education: Effects of a seven-month intervention. European Physical Education Review, 14(3), 367-387. http://pebites.org/download/i/mark_dl/u/4007149133/4539037722/ European\%252520Physical\%252520Education\%252520Review2008-Barkoukis-367-87.pdf

4. Barkoukis, V., Koidou, E., y Tsorbatzoudis, H. (2010). Effects of a motivational climate intervention on state anxiety, self-efficacy, and skill development in physical education. European Journal of Sport Science, 10(3), 167-177.

5. Carreiro da Costa, F. (1989). Estudo das condiçoes e factores de ensino-aprendizagem associados ao êxito numa unidade de ensino em educaçao física. Motricidad Humana. Lisboa: Ed. Facultad de Motricidad Humana.

6. Campbell, D. T., y Stanley, J. C. (1966). Experimental and Quasi-experimental Designs for Research. Nueva York: Rand McNally \& Company.

7. Conroy, D. E., Coastworth, J. D., y Kaye, M. P. (2007). Consistence of fear of failure score meanings among 8 to 18 year old female athletes. Educational and Psychological Measurement, 67(2), 300-310.

8. Conroy, D. E., Willow, J. P., y Metzler, J. N. (2002). Multidimensional fear of failure measurement: The performance failure appraisal. Journal of Applied Sport Psychology, 14, 76-90.

9. Conroy, D. E., Poczwardowski, A., y Henschen, K. P. (2001). Evaluative criteria and consequences associated with failure and success for elite athletes and performing artist. Journal of Applied Sport Psychology, 13, 300-322.

10. Deci, E. L., y Ryan, R. M. (1985). Intrinsic motivation and self-determination in human behavior. New York: Plenum.

11. Deci, E. L., y Ryan, R. M. (2000). The "what" and "why" of goal pursuits: Human needs and the self-determination of behaviour. Psychological Inquiry, 11, 227-268.

12. Digelidis, N., Papaioannou, A., Laparidis, K., y Christodoulidis, T. (2003). A One-year Intervention in 7th Grade Physical Education Classes Aiming to Change Motivational Climate and Attitudes toward Exercise. Psychology of Sport and Exercise, 4, 195-210.
13. Ferrer-Caja, E., y Weiss, M. R. (2000). Predictors of intrinsic motivation among adolescent students in physical education. Research Quarterly for Exercise and Sport, 71, 267-279.

14. Florence, J. (1991). Tareas significativas en Educación Física Escolar. Barcelona: INDE.

15. García-Calvo, T., Sánchez, P. A., Leo, F. M., Sánchez, D., y Amado, D. (2011). Incidencia de la Teoría de Autodeterminación sobre la persistencia deportiva. Revista Internacional de Ciencias del Deporte, 25(7), 266-276. Doi:10.5232/ricyde2011.02502. h

16. González-Cutre, D., Sicilia, A., y Moreno, J. A. (2011). Un estudio cuasi-experimental de los efectos del clima motivador tarea en las clases de educación física. Revista de Educación, 356, 677-700. http://www.mecd.gob.es/dctm/revista-de-educacion/articulosre356/ re35628.pdf?documentId=0901e72b81203178

17. Goudas, M., Biddle, S. J. H., y Fox, K. (1994). Perceived locus of causality, goal orientations and perceived competence in school physical education classes. British Journal of Educational Psychology, 64, 453-463.

18. Goudas, M., Biddle, S. J. H., Fox, K., y Underwood (1995). It ain't what you do, it's the way you do it! teaching style affects children's motivation in track and field lessons. The Sport Psychologist, 9, 254-264.

19. Halliburton, A. L., y Weiss, M. R. (2002). Sources of competence information and perceived motivational climate among adolescent female gymnasts varying in skill level. Journal of sport and exercise psychology, 24, 396-419.

20. Koka, A., y Hein, V. (2001). An impact of the teacher's feedback and perceived learning environment on intrinsic motivation among students involved and not involved in sport. Kehakuktuuriteaduskonna Teadus Ja Oppemetoodiliste Toode Kogumik, 9, 92-101.

21. Koka, A., y Hein, V. (2003). Perceptions of teacher's feedback and learning environment as predictors of intrinsic motivation in physical education. Psychology of Sport and Exercise, 4, 333-346. http://uuslepo. it.da.ut.ee/-andre/KokaHein(PSE).pdf

22. Koka, A., y Hein, V. (2005). The effect of perceived teacher feedback on intrinsic motivation in physical education. International Journal of Sport Psychology, 36(2), 91-106. http://kodu.ut.ee/-andre/ KokaHein(IJSP).pdf

23. Krane, V., Greenleaf, C. A., y Snow, J. (1997). Reaching for gold and the price of glory: A motivational case study of an elite gymnast. The sport psychologist, 11, 53-71.

24. Molina, L., Torres, E., y Miranda, M. T. (2008). El feed-back en educación física desde la perspectiva de género: Relación con la satisfac- 
ción en el área y los hábitos de actividad física extraescolar. Habilidad Motriz, 30, 5-22.

25. Moreno, J. A., y Cervelló, E. (2003). Pensamiento del alumno hacia la Educación Física: su relación con la práctica deportiva y el carácter del educador. Enseñanza, 21, 345-362. http://rca.usal.es/index.php/02125374/article/download/4068/4092

26. Moreno, J. A., Cervelló, E., y González-Cutre, D. (2007). Analizando la motivación en el deporte: un estudio a través de la teoría de la autodeterminación. Apuntes de Psicología, 1(25), 35-51.

27. Moreno-Murcia, J. A., y Conte, L. (2011). Predicción del miedo a equivocarse en jugadores de baloncesto a través del clima tarea de los iguales y la motivación intrínseca. Revista Mexicana de Psicología, 28(1), 43-52. http://www.redalyc.org/articulo.oa?id=243029630004

28. Moreno-Murcia, J. A., Conte, L., Silveira, Y., y Ruíz, L. M. (2014). Miedo a Fallar en el deporte. Editorial: Universidad Miguel Hernández de Elche. ISBN: 978-84-16024-10-0

29. Moreno, J. A., Gómez, A., y Cervelló, E. (2010). Un estudio del efecto de la cesión de autonomía en la motivación sobre las clases de educación física. Motricidad. European Journal of Human Movement, 24, 1-21.

30. Moreno, J. A., González-Cutre, D., y Chillón, M. (2009). Preliminary validation in Spanish of a scale designed to measure motivation in physical education classes: the Perceived Locus of Causality (PLOC) Scale. The Spanish Journal of Psychology, 12, 327-337.

31. Moreno, J. A., Hernández, A., y González-Cutre, D. (2009). Complementando la teoría de la autodeterminación con las metas sociales: un estudio sobre la diversión en educación física. Revista Mexicana de Psicología, 26(2), 213-222.

32. Moreno-Murcia, J. A., y Huéscar, E. (2012). Relación del tipo de feedback del docente con la percepción de autonomía del alumnado en clases de educación física. Infancia y aprendizaje, 35(1), 87-98.

33. Moreno-Murcia, J. A., Huéscar, E., y Cervelló, E. (2012). Predicción de la práctica física de los adolescentes al terminar la Educación Secundaria a partir de la teoría de la autodeterminación. The Spanish Journal of Psychology, 15, 90-100.

34. Moreno-Murcia, J. A., Huéscar, E., Peco, N., Alarcón, E., y Cervelló, E. (2013). Relación del feed-back y las barreras de comunicación del docente con la motivación intrínseca de estudiantes adolescentes de educación física. Anales de psicología, 29, 257-263. http://scielo.isciii. es/scielo.php?pid=S0212-97282013000100030\&script=sci_arttext

35. Moreno, J. A., y Llamas, L. S. (2007). Predicción de la importancia concedida a la educación física según el clima motivacional y la motivación autodeterminada en estudiantes adolescentes. Enseñanza, 25, 137-155. http://www.um.es/univefd/preef.pdf

36. Moreno-Murcia, J. A., Silveira, Y., Conte, L. (2013). Relación del feedback positivo y el miedo a fallar sobre la motivación intrínseca. Revista Española de Orientación y Psicopedagogía (REOP), 24(2), pp. 8-23 (ISSN electrónico: 1989-7448) www.uned.es/reop/pdfs/2013/24-2\%20completo.pdf

37. Moreno-Murcia, J. A., Zomeño, T., Marín, L. M., Ruiz, L. M., y Cervelló, E. (2013). Percepción de la utilidad e importancia de la educación física según la motivación generada por el docente. Revista de Educación, 362. doi: 10.4438/1988-592X-RE-2011-362-165. http://www. mecd.gob.es/dctm/revista-de-educacion/articulosre362/re36214. pdf?documentId=0901e72b816fbabc

38. Morgan, K., y Carpenter, P. (2002). Effects of Manipulating the Motivational Climate in Physical Education Lessons. European Physical Education Review, 8, 207-229.
39. Nicaise, V., Cogerino, G., Bois, J., y Amorose, A. J. (2006). Students' perceptions of teacher feedback and physical competence in physical education classes: Gender effects. Journal of Teaching in Physical Education, 25(1), 36-57.

40. Ntoumanis, N., y Biddle, S. J. H. (1999). A review of motivational climate in physical activity. Journal of Sports Sciences, 17, 643-665.

41. Ommundsen, Y. (2001). Pupils' affective responses in physical education classes: the association of implicit theories of the nature of ability and achievement goals. European Physical Education Review, 7, 219 242.

42. Silveira Torregrosa, Yolanda. Miedo a equivocarse en Educación Fisica y Deporte. Dirigida por Juan Antonio Moreno Murcia. Tesis doctoral inédita. Universidad Miguel Hernández de Elche. Departamento de Psicología de la Salud, 2013. http://dspace.umh.es/bitstream/11000/1366/1/TESIS\%20DOCTORAL\%20YOLANDA\%20 SILVEIRA\%20TORREGROSA.pdf

43. Smith, R., Smoll, F., y Hunt, E. (1977). A system for the behavioral assessment of athletic Coaches. Research Quarterly, 48, 401-407.

44. Solmon, M. A. (1996). Impact of motivational climate on students' behaviors and perceptions in a physical education setting. Journal of Educational Psychology, 88, 731-738.

45. Standage, M., Duda, J. L., y Ntoumanis, N. (2003). A model of contextual motivation in physical education: Using constructs from selfdetermination and achievement goal theories to predict physical activity intentions. Journal of Educational Psychology, 95, 97-110.

46. Theeboom, M., De Knop, P., y Weiss, M. R. (1995). Motivational climate, psychosocial responses, and motor skill development in children's sport: a field based-intervention study. Journal of Sport \& Exercise Psychology, 17, 294-311.

47. Treasure, D. C. (1993). A social-cognitive approach to understanding children's achievement behavior, cognitions, and affect in competitive sport. Unpublished doctoral dissertation, University of Illinois at Ur bana-Champaign.

48. Treasure, D. C., y Roberts, G. C. (2001). Students' perceptions of the motivational climate, achievement beliefs, and satisfaction in physical education. Research Quartely for Exercise and Sport, 2, 165-175.

49. Vallejo, G., y Plested, M. C. (2008). Tipos de comunicación en interacciones deportivas. Íkala, 13(19), 43-61.

50. Vallerand, R. J. (2007). Intrinsic and Extrinsic Motivation in Sport and Physical Activity. A Review an Look at the Future. En G. Tenenbaum, y R. C. Eklund (Eds.), Handbook of Sport Psychology (3a ed., pp. 59-83). New York: John Wiley.

51. Viciana, J., Cervelló, E., Ramírez, J., San-Matías, J., y Requena, B. (2003). Influencia del feed-back positivo y negativo en alumnos de secundaria sobre el clima ego-tarea percibido, la valoración de la EF y la preferencia en la complejidad de las tareas de clase. Motricidad. European Journal of Human Movement, 10, 99-116.

52. Wallhead, T. L., y Ntoumanis, N. (2004). Effects of a sport education intervention on students' motivational responses in physical education. Journal of Teaching in Physical Education, 23, 4-18.

53. Wilson, P. M., Rogers, W. T., Rodgers, W. M., y Wild, T. C. (2006). The Psychological Need Satisfaction in Exercise Scale. Journal of Sport and Exercise Psychology, 28, 231-251. http://sdtheory.s3.amazonaws. com/SDT/documents/2006_WilsonRogersWild_JSEP.pdf

54. Zuckerman, M., y Tsai, F. (2005). Costs of self-handicapping. Journal of Personality, 73, 411-442. 